\title{
Health-related Quality of Life and Leisure Participation among Long-term Care Facility Residents with Stroke
}

Pei-Shan Wu ${ }^{1}$, Hui-Man Huang ${ }^{2 *}$, Wan-Ju Lin ${ }^{3}$ and Tzu-Chi Chen ${ }^{4}$

${ }^{1}$ Instructor, Department of Nursing, Chung Hwa University of Medical Technology, Taiwan

${ }^{2}$ Associate Professor, Department of Nursing, National Quemoy University, Kinmen County, Taiwan

${ }^{3}$ Leader, Emergency Department, Tainan Sin-Lau Hospital, Taiwan

${ }^{4}$ Department of Nursing, National Quemoy University, Taiwan

\section{Abstract}

Publication History:

Background: Long-term care facility residents with stroke typically engage in daily life activities and leisure activities with low frequency, which may affect their health-related quality of life (HRQoL).

Purpose: The current study was conducted to (1) Explore stroke residents' leisure-activity participation and HRQoL, as well as related factors, and (2) Investigate the relationship between stroke residents' leisureactivity participation and HRQoL.

Methods: A cross-sectional correlational research design with convenience sampling was applied to select 70 individuals with stroke living in long-term care facilities in Taiwan. The questionnaire included items regarding the residents' characteristics, self-perceived health status, activities of daily living (ADLs), and leisure-activity participation frequency, as well as the Stroke Impact Scale (SIS).

Results: (1) The female residents had a higher frequency of active leisure-activity participation than the male residents. (2) Self-perceived health status was significantly and positively correlated with the overall frequency of leisure-activity participation, static leisure activities, and active leisure activities, while ADLs were significantly and positively correlated with the overall frequency of leisure-activity participation, static leisure activities, active leisure activities, and periodic routine activities. (3) A significant positive correlation was found between overall HRQoL and ADLs, the overall frequency of leisure-activity participation, static leisure activities, and active leisure activities.

Conclusions: The relationship between leisure-activity participation and HRQoL was identified. In order to improve the HRQoL of long-term care facility residents with stroke, it is suggested that staff members should provide individualized activities to enhance residents' abilities to engage in ADLs and increase their participation in leisure activities. Further studies are needed to reveal whether improvement of leisureactivity participation could affect HRQoL in various dimensions.

\section{Introduction}

Suffering from a stroke causes physical and psychological deficits that affect an individual's activities of daily living (ADLs), which leads in turn to changes in the patterns of daily living and further impacts his or her quality of life (QoL) [1,2]. Kranciukaite and Rastenyte [3] emphasized that the goal of therapy in stroke treatment has shifted from the traditional approach of focusing on maintaining and restoring physical functions to improving the overall QoL of an individual Chiou [4] pointed out that $6.4 \%$ of stroke patients in Taiwan enter institutions for long-term care after they were discharged. The ADLs of the residents in these institutions are reduced due to their illnesses and their limited ability to participate in activities. This results in the residents being confined to their beds or wheelchairs during the daytime except for when dining and sleeping. Moreover, the lack of activities provided by institutional medical personnel and caregivers to residents and the lack of equipment and space for the activities within the institution are the two main reasons leading to the further rapid deterioration of the physical functions of institutional residents [5]. In particular, stroke residents living in small-scale institutions lack opportunities to properly participate in activities [6,7], which affects the QoL of these stroke residents.

\section{Literature Review}

\section{Stroke residents in long-term care facilities}

The impact of suffering a stroke on an individual is multi-faceted. The clinical manifestations of stroke are sudden local nerve defects, which cause limb hemiplegia, dysphagia, dysarthria, or gait instability. The stroke is a chronic disease that affects the physiological, psychological, social, and cognitive aspects of the patient. Physical activity dysfunction and emotional reactions also affect the original functions of daily life [8]. The range of personal function limitations caused by stroke includes physical paralysis, the impairment of sensory movement and cognition, and speech or communication difficulties, and these limitation hamper interpersonal interactions and the social roles of the patients. Chiou's [4] research showed that after stroke patients are discharged from hospital, only $32.2 \%$ of them are completely independent in terms of their ADLs. Furthermore, $22.6 \%$ of such patients have moderate or even severe dependence, and $6.4 \%$ require long-term care. Taiwan's long-term care facilities refer to nursing homes, elder care and rehabilitation centers, long term care facilities and Ren-ai senior citizen's homes that are part of the social affairs system and were chartered by the government. According to the statistical data of the Department of Health and Welfare, Statistics

"Corresponding Author: Dr. Hui-Man Huang, Department of Nursing, National Quemoy University No. 1, University Rd. Jinning Township, Kinmen County 89250, Taiwan, Tel: + 886-82-313717;0956070508, Fax: +886-82-313-444; E-mail: tel7222662@gmail.com

Citation: Wu PS, Huang HM, Lin WJ, Chen TC (2019) Health-related Quality of Life and Leisure Participation among Long-term Care Facility Residents with Stroke. Int J Nurs Clin Pract 6: 313. doi: https://doi.org/10.15344/2394-4978/2019/313

Copyright: (C) 2019 Wu et al. This is an open-access article distributed under the terms of the Creative Commons Attribution License, which permits unrestricted use, distribution, and reproduction in any medium, provided the original author and source are credited. 
Department [9], stroke accounted for the highest proportion (44\%) of the diseases that affected residents in Taiwan's long-term care facilities. Furthermore, the stroke residents in long-term care facilities are mostly in the chronic phase of stroke.

\section{Health-related quality of life in stroke cases}

The World Health Organization (WHO) defines QoL as an individual's perception of their position in life in the context of the culture and value systems in which they live and in relation to their goals, expectations, standards, and concerns. It is a broad ranging concept affected in a complex way by the person's physical health, psychological state, degree of independence, personal beliefs, and social relationships, as well as their relationship to salient features of their environment $[10,11]$. The QoL of an individual is influenced by many factors. An individual's overall QoL can be assessed in terms of an individual's subjective cognitive level, positive or negative emotions, and physical and mental health [12]. Lee [13] advocates assessing an individual's QoL based on the individual's performance in physical, mental, and interpersonal relationships. For example, from the physical perspective, whether an individual can act on his own and whether he needs assistive devices or other people's assistance are indicators that can be used in assessing the QoL of an individual This kind of assessment has a certain objective meaning. It assesses whether the patient is affected by the disability and whether his/her QoL is restricted due to the disability. This assessment also applies to the situation in terms of psychological and social aspects.

When the care provided to a stroke patient gradually shifts to supportive medical care because of chronic illness or disability, the improvement of health-related QoL (HRQoL) has become the main goal of the care service. Therefore, scholars have put forward the assessment of HRQoL in the hopes of understanding the impact of stroke on the individual and making objective assessments. Lin et al. [1] discussed the HRQoL of 124 stroke patients and found that the patients' age, gender, educational level, marital status, disease duration, and disease severity were significantly correlated with HRQoL. Li, Wang, and Lin [14] pointed out that the religious belief of stroke patients, which was one of the basic attributes that they examined, was related to HRQoL. Yang et al. [15] pointed out that physical aging or disease will reduce the ADL. The lower ADLs are not only highly dependent, but also indicate that their quality of life is affected. Lee, $\mathrm{Wu}, \mathrm{Chiu}, \mathrm{Twu}$, and Hsieh [16] found a significant association between ADLs function and HRQoL in patients with strokes. Meanwhile, stroke residents living in long-term care facilities suffer physiological sequelae such as hemiplegia and hypodynamia, so they show different degrees of dependence in terms of daily life functions, which in turn affects their QoL.

\section{Participation in leisure activities}

Lee and Chen [17] defined "leisure activities" to include a multifaceted range of activities such as sports, games, travel, and social activities that an individual initiates and participates in under his or her own volition. "Participation in leisure activities" refers to an individual choosing these types of non-work related activities of his/her own free will in order to satisfy his/her own psychological or physical desires [18], and it also refers to the frequency of an individual engaging in certain leisure activities [19], It is known from the aforementioned definitions that participating in leisure activities has the positive effects of helping physical and mental development and enriching life. Riddick and Stewart [20], Iso-Ahola [21], and Zeng
[22] advocated that participating in leisure activities can bring physical and mental health and life satisfaction to an individual. In addition, through the process of participating, interpersonal relationships will be maintained and communication skills will be increased. Participating in leisure activities also helps an individual rebuild his/ her daily life and maintain ADLs capabilities. Domestic research has indicated that individual basic attributes, ADLs, and self-perceived health status affect the frequency of an individual's participation in leisure activities. Zheng [23] studied the participation in leisure activities of elderly people living alone in Taiwan and found that there were statistically significant correlations and differences in their level of participation in leisure activities due to gender, age, educational level, chronic disease status, and ADL ability. Kao, Lin, Huang, and Lin [24] studied 176 elderly people who participated in community care center activities and found that those with high self-perceived health status engage in more leisure activities.

\section{Relationship between leisure-activity participation and HRQoL}

Leisure field includes sports, games and physical activity etc. Yang [25] studied adults in Taipei City and found that the total amount of individual participation in leisure activities was significantly related to QoL. Leisure activities are one of the types of physical activity. Yen and Chen [26] discussed the amount of physical activities engaged in by the elderly in long-term care facilities, emphasizing that physical activities can reduce life or illness-induced pressure on individuals and have positive effects on their physical and mental health. It has also been deduced that participation in leisure activities can strengthen individuals' identities and enhance their satisfaction with life, further increasing their QoL. However, research regarding the relationship between the HRQoL of stroke patients and the frequency of their participation in leisure activities is lacking. At present, in Taiwan, research regarding stroke patients rarely focuses on their leisure activity participation. Some research focuses on medical care, rehabilitation, or care services. For example, recent studies have focused on confirming the factors that affect the QoL [27], understanding the consistency of QoL in stroke patients and their caregivers [28], and exploring the HRQoL of outpatients with chronic stroke and related factors [14]. There is also a relative lack of research on the relationship between HRQoL and the participation in leisure activities of residents in long-term care facilities. Therefore, this study selected stroke residents in long-term care facilities as its subjects and further discussed: (1) The frequency of their participation in leisure activities, their HRQoL, and related factors and (2) The correlation between their participation in leisure activities and HRQoL. The results of the study can provide a reference for caregivers of long-term care facilities when designing leisure activities aimed at improving the HRQoL of stroke residents.

\section{Methods}

This study adopted a cross-sectional correlational study design to collect information from stroke residents staying in long-term care facilities, conducting face-to-face interviews from September 21, 2015, to January 20, 2016.

\section{Subject}

Seventy stroke residents in 20 long-term care facilities in Taiwan were selected as the subjects using the convenience sampling method. To be enrolled, an individual had to meet the following inclusion criteria: (1) Be over 20 years old and a member of the long-term care 
facility who had been diagnosed by a physician as a stroke patient. These residents are diagnosed with a stroke by a physician, whether it is a hemorrhagic or ischemic stroke; (2) Have the ability to clearly communicate; and (3) Have daily life functioning anywhere between independent and moderately dependent (i.e., ADLs scores of 61-100), The number of participants in this research was calculated according to Cohen's [29] Bivariate Correlation Coefficients. The significance level was set at $\alpha=.05$, the statistical power was set as 0.8 , the effect size was 0.15 , and Version 3.1 G-power was used to estimate the number of samples required for this study. The number of subjects recruited was 70 . The power from examining that number of samples reaches 0.8 , indicating that this study has acceptable power.

\section{Instruments}

\section{Resident basic attribute questionnaire}

The questionnaire included items regarding the age, gender, marital status, educational level, number of years since onset, self-perceived health status, and ADLs of the respondent. There were two items regarding self-perceived health status: (1) What do you think of your current physical health condition? And (2) How you feel about the state of your health compared to a year ago. After adding up the scores of the two questions, the total scores are between $0-4$. The higher the scores, the better the self-perceived health status is. As for the ADLs, they were calculated according to the Barthel Index scores within a month, with the total scores being from 0-100. The higher the scores, the higher the ADLs, and the better the mobility is. Specifically, a score of 0-20 indicates complete dependence; 21-60 indicates severe dependence; 61-90 indicates moderate dependence; and 100 indicates complete independence.

\section{Leisure activity participation scale}

The researcher adapted the "Recreation Activity Participation Scale" of Lee, Shih, and Chiou [30] as a reference and drafted a related scale for research regarding residents' participation in leisure activities. The scale comprised 26 items regarding leisure activities, including items about static activities ( 8 items), dynamic activities (10 items), regular activities held by the long-term care facilities ( 7 items), and others (1 item). The scoring method was based on Kao's [31] absolute frequency method in order to compile the statistics regarding the frequency of residents' participation in the leisure activities. Each resident marked down the number of times they had participated in the activities in the past week, with one point for one act of participation in a week, two points for two times, and so on. The total frequency range was from 0 to 182 points. The higher the score, the higher the total frequency of leisure activity participation are. In terms of total score, the score ranges for static activities, dynamic activities, and regular activities (held by long-term care facilities) were 0 to 56,0 to 70 , and 0 to 56 , respectively. The average score for each type of leisure activity participation was calculated by dividing the various types of leisure activities by the number of the questions to rank the frequency of participation in the different types of leisure activities. The higher the score, the higher the participation frequency was. Li [32] previously adopted this scale to discuss the leisure activity participation of senior citizens in nursing homes. The measured Cronbach's a value of the scale is .75, and it is known that this scale has acceptable credibility.

\section{The stroke impact scale (SIS)}

This study adopted the Taiwan Version of the Stroke Impact Scale (Version 3.0) translated and developed by Chung [33] as the tool to measure the HRQoL of the residents. The scale covers eight aspects via 59 questions: strength (4 questions); memory (7 questions); emotions (9 questions); communication (7 questions); mobility (10 questions); ADLs/IADL (9 questions); hand function (5 questions); and participation (8 questions). Each item of the scale is scored using a five-point Likert scale. The score of each aspect is adjusted according to [(average score-1)/(5-1)] $\times 100$, and the total score is between 0 to 100 points. Three questions regarding the aspect of emotions are reverse-scored items. The higher the total score, the better the overall HRQoL as perceived by the given stroke resident is. Liang and Lin [34] used the SIS to measure the QoL of 102 patients with stroke in Taiwan and confirmed that the SIS has good reliability and validity. After the measurements in this study were fully conducted, the Cronbach's a coefficient that measures the internal consistency of the SIS was found to be .96 , indicating that this scale has acceptable reliability.

\section{Ethical considerations}

This research was reviewed and approved by an Institutional Review Board (IRB) (Jianan No. 15-025). Before the interview, the researcher introduced herself to the given resident; explained the research purpose and process, as well as the participant's rights; and then obtained informed consent from the resident. The data provided by the research subjects will be used only for academic purposes and will not be publicly released. Moreover, the data analysis was not conducted with regard to the data of any individual; instead, it only expressed opinions of the full study group.

\section{Data analysis}

In this study, the SPSS20.0 for Windows statistical software suite was used for data processing and analysis. The descriptive statistics were calculated with percentage, average, and standard deviation, and the inferential statistics were calculated using the $t$-test, oneway ANOVA, and Pearson product-moment correlation coefficient analysis.

\section{Results}

\section{Basic attributes of residents, participation in leisure activities and HRQoL}

Table 1 shows that the average age of the residents is $73.56 \pm 10.20$ years old, the majority of the residents are $65-80$ years old $(61.45 \%)$, males $(64.3 \%)$, married $(67.1 \%)$, went to or graduated from elementary schools $(42.9 \%)$. The average number of years of stroke onset is $5.85 \pm 4.85$ years, and the self-perceived health status of the residents is $1.96 \pm 1.16$ points, which is classified as "lower degree". The average score of ADLs is $74.86 \pm 13.65$ points, which means "moderate dependence", and the majority of the residents are moderately dependent (78.6\%). Table 2 shows that the residents' overall frequency of leisure-activity participation is at a low level (an average of $21.21 \pm 11.11$ points); static activities show the highest frequency of leisure-activity participation, with an average of $11.40 \pm 6.28$. Table 3 shows that the overall HRQoL of the residents is at a normal level, with an average of $60.20 \pm 15.52$ points; in terms of scores on all levels of HRQoL, the "communication" ability shows the best result (an average of $91.17 \pm 11.68$ points).

\section{The relationship between basic attributes, and participation in leisure activities and HRQoL}

Table 4 shows that the participation frequency of female residents in dynamic leisure activities is significantly higher than that of men 
Citation: Wu PS, Huang HM, Lin WJ, Chen TC (2019) Health-related Quality of Life and Leisure Participation among Long-term Care Facility Residents with Stroke. Int J Nurs Clin Pract 6: 313. doi: https://doi.org/10.15344/2394-4978/2019/313

Page 4 of 7

$(t=-2.45, p<.05)$. There is a significant positive correlation among the self-perceived health status and the total frequency of participation $(r=.35, p<.01)$, static leisure activities $(r=.28, p<.05)$ and dynamic leisure activities $(r=.30, p<.05)$ of the residents. There is a significant positive correlation among the residents' ADLs and the total frequency of participation $(r=.46, p<.001)$, static leisure activities $(r=.35, p<.01)$, dynamic leisure activities $(r=.41, p<.001)$ and regular activities held by long-term care facilities $(r=.25, p<.05)$. There is an obvious positive correlation between residents' ADLs and overall HRQoL scores $(r=.80, p<.001)$. This means the better the ADLs are, the higher their HRQoL is.

\begin{tabular}{|c|c|c|}
\hline Variables & $\mathrm{n}(\%)$ & $\mathrm{M} \pm \mathrm{SD}$ \\
\hline \multicolumn{2}{|l|}{ Age (years old) } & $73.56 \pm 10.20$ \\
\hline $50-64$ & $12(17.1)$ & \\
\hline $65-80$ & $43(61.45)$ & \\
\hline $81-94$ & $15(21.4)$ & \\
\hline \multicolumn{3}{|l|}{ Gender } \\
\hline Male & $45(64.3)$ & \\
\hline Female & $25(35.7)$ & \\
\hline \multicolumn{3}{|l|}{ Marital Status } \\
\hline Married & $47(67.1)$ & \\
\hline Not married & $23(32.9)$ & \\
\hline \multicolumn{3}{|l|}{ Educational Level } \\
\hline Illiterate & $14(20.0)$ & \\
\hline Went to or graduated from elementary school & $30(42.9)$ & \\
\hline Went to or graduated from junior high school & $15(21.4)$ & \\
\hline Went to or graduated from high school & $8(11.4)$ & \\
\hline College or above & $3(4.3)$ & \\
\hline \multicolumn{2}{|l|}{ Number of Years since Onset } & $5.85 \pm 4.85$ \\
\hline \multicolumn{2}{|l|}{ Self-Perceived Health Status } & $1.96 \pm 1.16$ \\
\hline \multicolumn{2}{|l|}{ ADLs } & $74.86 \pm 13.65$ \\
\hline Moderate dependence & $55(78.6)$ & \\
\hline Mild dependence & $9(12.9)$ & \\
\hline Complete independence & $6(8.5)$ & \\
\hline
\end{tabular}

\begin{tabular}{|l|l|l|l|}
\hline Variables & $\mathrm{M} \pm \mathrm{SD}$ & Single Item Average Score & Rank \\
\hline Overall Participation Frequency (total scores 182) & $21.21 \pm 11.11$ & & \\
\hline Static Activities (total scores 56) & $11.40 \pm 6.28$ & 1.43 & 1 \\
\hline Dynamic Activities (total scores 70) & $9.01 \pm 6.54$ & 0.90 & 2 \\
\hline Regular Activities (total scores 56) & $0.80 \pm 1.16$ & 0.1 & 3 \\
\hline
\end{tabular}

Table 2: Score distribution of the residents' leisure activity participation frequency $(\mathrm{N}=70)$.

\begin{tabular}{|l|l|l|l|}
\hline Quality of Life Aspect & $\mathrm{M} \pm \mathrm{SD}$ & Score Range & Rank \\
\hline Overall Quality of Life & $60.20 \pm 15.52$ & $29.24-96.19$ & \\
\hline strength & $34.38 \pm 25.75$ & $0-93.75$ & 8 \\
\hline memory & $83.47 \pm 17.38$ & $28.57-100$ & 2 \\
\hline emotion & $66.87 \pm 20.33$ & $27.78-100$ & 3 \\
\hline communication & $91.17 \pm 11.68$ & $46.43-100$ & 1 \\
\hline ADLs/IADL & $59.82 \pm 21.31$ & $27.50-100$ & \\
\hline mobility & $49.56 \pm 27.54$ & $11.11-100$ & \\
\hline hand function & $41.71 \pm 34.10$ & $0-100$ & \\
\hline participation & $42.14 \pm 21.20$ & $0-100$ & \\
\hline
\end{tabular}


Citation: Wu PS, Huang HM, Lin WJ, Chen TC (2019) Health-related Quality of Life and Leisure Participation among Long-term Care Facility Residents with Stroke. Int J Nurs Clin Pract 6: 313. doi: https://doi.org/10.15344/2394-4978/2019/313

Page 5 of 7

\begin{tabular}{|l|l|l|l|l|l|}
\hline Variables & $\begin{array}{l}\text { Overall Participation Frequency } \\
\text { of Leisure Activity }(t / r)\end{array}$ & $\begin{array}{l}\text { Static Activities } \\
(t / r)\end{array}$ & $\begin{array}{l}\text { Dynamic Activities } \\
(t / r)\end{array}$ & $\begin{array}{l}\text { Regular Activities } \\
(t / r)\end{array}$ & $\begin{array}{l}\text { Overall HRQoL } \\
(t / r)\end{array}$ \\
\hline Gender & -1.53 & -0.08 & $-2.45^{*}$ & 0.24 & 1.19 \\
\hline Male & $19.69 \pm 10.74$ & $11.36 \pm 6.19$ & $7.51 \pm 5.40$ & $0.82 \pm 1.30$ & \\
\hline Female & $23.96 \pm 11.47$ & $11.48 \pm 6.57$ & $11.72 \pm 7.59$ & $0.76 \pm 0.88$ & \\
\hline Self-Perceived Health Status & $.35^{* *}$ & $.28^{*}$ & $.30^{*}$ & .08 & .104 \\
\hline ADLs & $.46^{* * *}$ & $.35^{* *}$ & $.41^{* * *}$ & $.25^{*}$ & $.000^{* * *}$ \\
\hline
\end{tabular}

Table 4: Breakdown of differences and correlations between leisure activity participation and HRQoL according to the residents' attributes ( $\mathrm{N}=70$ ). ${ }^{*} p<.05,{ }^{* *} p<.01,{ }^{* *} p<.001$

The correlation between participation of residents in leisure activities \& HRQoL

Table 5 shows there is an evident positive correlation among the overall HRQoL of the residents and the total frequency of leisure activity participation $(r=.50, p<.001)$, participation frequency of static activities $(r=.49, p<.001)$, and participation frequency of dynamic activities $(r=.34, p<.01)$. In other words, the higher the overall participation frequency, the higher the participation frequency of static activities, and the higher the participation in dynamic activities, the better the residents' HRQoL.

\begin{tabular}{l|l|}
\hline Variables & Overall HRQoL $(t / r)$ \\
\hline Overall Participation Frequency & $.50^{* * *}$ \\
\hline Static Activity Participation Frequency & $.49^{* * *}$ \\
\hline Dynamic Activity Participation Frequency & $.34^{* *}$ \\
\hline Regular Activity Participation Frequency & .22 \\
\hline $\begin{array}{l}\text { Table 5: Correlations between leisure activity participation and } \\
\text { HRQoL }(\mathrm{N}=70) .\end{array}$ \\
$* * 0.01,{ }^{* *} p<.001$
\end{tabular}

\section{Discussion}

The relationship between basic attributes of the residents and participation in leisure activities

Leisure is viewed as a fundamental component of life. Although there are many different types of leisure activities, one of the key elements to leisure activities is its relationship to the individual's health [38]. The results of this study show that the participation frequency of dynamic leisure activities of female residents is significantly higher than that of men. This is similar to Zheng's [23] finding that the participation frequency of gender of the elderly living alone is statistically and significantly different. However, Tsai [35] explored the motivation and participation of 297 stroke patients in leisure activities, and found that there was no difference in participation types and frequency in terms of different genders. The results of this study show a correlation between being female and having a higher frequency of participation in dynamic leisure activities, so it is worth studying in the future to obtain equality between different genders. Moreover, the results show that the residents with better self-perceived health status are keen to participate in leisure activities, static activities, and dynamic activities, which is similar to the results of Kao et al. [24]. In addition, the better the residents' ADLs, the higher their overall participation frequency, participation frequency of static activities, dynamic activities, and regular activities are. This result is similar to that of Zheng's [23]. Furthermore, Tsai's [35] study also pointed out that the better the ADLs of the residents, the better the residents participate. It means that the residents can arrange and participate in various types of leisure activities which is organized by the long-term care facilities.
The relationship between the basic attributes of the residents and HRQOL

The better the ADLs of stroke residents, the better the HRQoL, which is similar to the findings of many studies on the QoL of patients with stroke [14,16,36,37]. The loss of ADLs' function reflects the obstacles of the residents' ability to self-care and requires care services. The residents with better ADLs have the ability to choose the lifestyle they want, so they can obtain better HRQoL.

\section{The relationship between the participation in leisure activities of the residents and HRQoL}

The results clearly show that both the overall participation frequency and dynamic leisure activities are positively correlated with the overall HRQoL. This is similar to Yang's [25] research results which suggested that individuals who participate in leisure activities more often have better QoL. In addition, the participation of the residents in static activities is also positively correlated with the overall HRQoL. This is similar to the results of $\mathrm{Li}$ et al. [14] concerning the status of leisure activities for 55-year-olds in the community. Li et al. [14] confirmed that there are positive influences on the elderly people in the community who participate in static leisure activities. The activity of the highest participation frequency for stroke residents of this study is "static activity". Participating in static leisure activities not only relieves the emotions of the stroke residents, but also increases their daily life mobility and socialization ability. As a result, the residents feel an increase in their QoL.

\section{Conclusion and Suggestion}

This study selected 70 residents of the long-term care facilities in Tainan as the subjects with the convenience sampling method. The results showed that: (1) The influencing factors related to the leisure activity participation of stroke residents include gender, self-perceived health status and ADLs; (2) The factors affecting the overall HRQoL of stroke residents include ADLs, overall frequency of leisure activity participation, static leisure activities, and dynamic leisure activities. In other words, the higher the ADLs, the higher the participation frequency of leisure activities, static leisure activities and dynamic leisure activities of the residents, the better the HRQoL is.

There are three limitations of this study: (1) The sampling method is convenience sampling method. The research subjects are limited to the residents in long-term care facilities in Tainan region. The sample cannot represent the group. Therefore, the result cannot be applied to all stroke residents in long-term care facilities around Taiwan. (2) One limitation is the relatively low number of participants in the study, and more long-term studies in different regions are needed to confirm our results. (3) The study subjects are conscious and able to communicate 
Citation: Wu PS, Huang HM, Lin WJ, Chen TC (2019) Health-related Quality of Life and Leisure Participation among Long-term Care Facility Residents with Stroke. Int J Nurs Clin Pract 6: 313. doi: https://doi.org/10.15344/2394-4978/2019/313

with Chinese and Taiwanese. The ADLs of majority of the residents are moderate dependence $(78.6 \%)$. The data of the participation in leisure activities and the HRQoL of the residents who are not able to communicate or whose ADLs are severely or completely dependent were not collected. This is the third limitation of this research.

In regards to the future research, the researcher suggested that: (1) Further studies with a larger sample are recommended for future consideration to refine and consolidate our findings; (2) Comparison and analysis of the differences in HRQoL among stroke residents under varying frequencies of participation in leisure activities; (3) To repeat the work in another country as well. In terms of caregivers of the long-term care facilities, it is recommended that they: (1) Increase the number of times that stroke residents participate in leisure activities. Even if the activities are static such as watching television, listening to music or reading newspapers, these activities can also increase their QoL; (2) Caregivers should help to improve residents' ADLs because higher performance of ADLs links to higher involvement in activities; and therefore an increased QOL; (3) For bed-ridden residents, caregivers can give a weekly reading of novels or newspapers at a fixed time on the bedside, or provide a bedside TV for the residents to watch when they are awake, and caregivers can also provide individual leisure activities to meet the mental and social needs of the stroked residents.

\section{Author Contributions}

Study conception and design: Pei-Shan Wu \& Hui-Man Huang

Data collection: Pei-Shan Wu

Data analysis and interpretation: Hui-Man Huang \& Pei-Shan Wu Drafting of the article: Wan-Ju Lin\& Hui-Man Huang\&Tzu-Chi Chen Critical revision of the article: Hui-Man Huang

\section{Competing Interests}

The authors declare that they have no competing interests.

\section{References}

1. Lin TH, Huang LY, Hung LC, Fan SH (2008) The first probe for stroke patient's health-related quality of life. Journal of Health Management 6: 121-134.

2. Muro MJ, Pedro-Cuesta J, Almazán J, Holmqvist LW (2000) Stroke patients in South Madrid: Function and moto recovery, resource utilization, and family support. Stroke 31: 1352-1359.

3. Kranciukaite $D$, Rastenyte $D$ (2006) Measurement of quality of life in stroke patients. Medicina 42: 709-716.

4. Chiou HY (2008) Current situation and epidemiological features of stroke. Stroke 15: 2-4.

5. Fuh BI, Lu HH, Chang KJ (2007) A project for improving activities of nursing home residents. The Journal of Long-term Care 11: 381-391.

6. Lin SC, Chen SM (2012) Assisting an old adult with stroke in developing regular exercise at a long-term care institution. The Journal of Long-term Care 16: 305-315.

7. Hou CY (2005) The function of activity therapy in long-term care institutions Taiwan Journal of Gerontological Health Research 1: 43-51.

8. Tsai HJ, Tseng SP, Wang SY, Chang YY, Chao YH (2016) Exploring the primary adaptation process of first-ever stroke patients. The Journal of Nursing 63: 103-112.

9. Department of Health and Welfare Statistics (2015) Long-term care of the elderly, maintenance and security institutions overview. Retrieved from www.mohw.gov.tw/CHT/DOS/DisplayStatisticFile.aspx?d=31859
10. The WHOQOL Group (1998) The World Health Organization Quality of Life assessment (WHOQOL): Development and general psychometric properties. Social Science Medicine 46: 1569-1585.

11. The WHOQOL Group (1998) Development of the World Health Organization WHOQOL-BREF quality of life assessment. Psychological Medicine 28: 551558.

12. Lin PS (2008) Stroke measurement of outcomes- quality of Life. Taiwan Stroke Society News 15: 7-9.

13. Lee RC (2012) Analysis of wellbeing: Quality of life. Applied Ethics Review 52: 141-152.

14. Li CH, Wang JY, Lin YJ (2012) Health-related quality of life and related factors among chronic stroke patients in rehabilitation outpatient clinics. The Journal of Long-term Care 16: 143-158.

15. Yang YA, Chen YS, Lin CF, Lee HC, Tsai YJ, et al. (2017) An investigation of ADL disability status and associated physical performances in mobile but unstable institutionalized older adults. Psychological Medicine 42: 135-136.

16. Lee YC, Wu ZI, Chiu EC, Twu FC, Hsieh CL (2012) Factors predicting overall health-related quality of life in patients with stroke. Journal of Occupational Therapy Association 30: 53-67.

17. Lee PC, Chen CT (2009) Leisure, leisure activities and leisure sports. Taichung University of Education Department of Physical Education 4: 48-57.

18. Chung GX, Lin J (2002) Leisure management. Taipei: Yang Zhi Culture.

19. Ragheb MG, Griffith CA (1982) To contribution of leisure participation and leisure satisfaction to life satisfaction of older persons. Journal of Leisure Research 14: 295-306.

20. Riddick CC, Stewart DG (1994) An examination of the life satisfaction and importance of leisure in the lives of older female retirees: A comparison of blacks to whites. Journal of Leisure Research 26: 75-87.

21. Iso-Ahola SE (1980) The social psychology of leisure and recreation. Dubuque, IA: Wm, Brown C Company Publisher

22. Zeng ZS (2012) Exploring the participation of leisure activities in older people. Leisure and Health 7: 111-119.

23. Zheng SW (2005) The effects of the leisure activities and social relationships on the psychological status of the elderly living alone. Retrieved from National Digital Library of Theses and Dissertations in Taiwan.

24. Kao CC, Lin PC, Huang HM, Lin YH (2013) The relationship between physical activity and quality of life in community-care-site older adults. Nursing and Health Care Research 9: 157-169.

25. Yang CT (2003) The relationship between leisure activity participation and quality of life in Taipei city. Retrieved from National Digital Libraryof Theses and Dissertations in Taiwan.

26. Yen ML, Chen YM (2008) Physical activity and related factors among older adults living in care institutions. Journal of Evidence-Based Nursing 4: 8998.

27. Liu HH, Lin PS, Liang PJ, Wang CM (2009) The predictors of 3-month quality of life outcomes for stroke patients in Taiwan. Formosan Journal of Physical Therapy 34: 53-64.

28. Cheng TC, Wang SH, Chiang HY, Shih YN, Ou YC, et al. (2012) Consistency of the stroke-specific health-related quality of life between self-reports and proxy assessments. Formosan Journal of Medicine 16: 230-235.

29. Cohen J (1988) Statistical Power Analysis for Behavioral Sciences (2nd Ed). Hillsdale NJ: Lawrence Erlbaum.

30. Lee WL, Shih JB, Chiou SL (2007) Leisure activity participation and happiness among the retired elderly in Kaohsiung. Journal of Humanities and Social Sciences 3: 27-35.

31. Kao CH (1999) A comparative study of athletes' leisure participation, leisure benefits and perceived training effects. Outdoor Recreation Research 12: 43-61.

32. $\quad \mathrm{Li} L(2007)$ The relationships between leisure participation and depression in the residents in LTC facilities-A recursive model. Retrieved from National Digital Library of Theses and Dissertations in Taiwan.

33. Chung RC (2004) Applying Stroke Impact Scale to explore the quality of life of stroke patients. Taipei City: National Science Council of the Executive Yuan.

34. Liang PJ, Lin PS (2016) Reliability and validity of the Stroke Impact Scale 3.0 in Taiwanese stroke patients. Formosa Journal of Physical Therapy 41: 28-36. 
Citation: Wu PS, Huang HM, Lin WJ, Chen TC (2019) Health-related Quality of Life and Leisure Participation among Long-term Care Facility Residents with Stroke. Int J Nurs Clin Pract 6: 313. doi: https://doi.org/10.15344/2394-4978/2019/313

Page 7 of 7

35. Tsai MG (2008) The participation motivation, participation and benefit of the daily-life and leisure activities for stroke patients. Retrieved from National Digital Library of Theses and Dissertations in Taiwan.

36. Kao CF, Chiang HL, Shih LC, Liu SJ (2008) Quality of life and related factors among stroke outpatients. Journal of Evidence-Based Nursing 4: 127-137.

37. Hsu JL (2010) The factors related to stroke rehabilitation and quality of life in patients with stroke. Retrieved from National Digital Library of Theses and Dissertations in Taiwan.

38. Chin MK, Edginton CR, Fleming KM, Flack TM, Ruan B (2013) Leisure, physical activity and community healthy living. International Leisure Review 2: 1-32. 